\title{
Publisher's Note: Sensitivity of the Advanced LIGO detectors at the beginning of gravitational wave astronomy [Phys. Rev. D 93, 112004 (2016)]
}

\author{
D. V. Martynov et al.
}

(Received 14 March 2018; published 20 March 2018)

DOI: 10.1103/PhysRevD.97.059901

This paper was published online on 2 June 2016 with an omission of eight author names in the author list. The author list should read as: D. V. Martynov, ${ }^{1}$ E. D. Hall, ${ }^{1}$ B. P. Abbott, ${ }^{1}$ R. Abbott, ${ }^{1}$ T. D. Abbott, ${ }^{2}$ C. Adams, ${ }^{3}$ R. X. Adhikari, ${ }^{1}$ R. A. Anderson, ${ }^{1}$ S. B. Anderson, ${ }^{1}$ K. Arai, ${ }^{1}$ M. A. Arain, ${ }^{4}$ S. M. Aston, ${ }^{3}$ L. Austin, ${ }^{1}$ S. W. Ballmer, ${ }^{5}$ M. Barbet, ${ }^{4}$ D. Barker, ${ }^{6}$ B. Barr, ${ }^{7}$ L. Barsotti ${ }^{8}$ J. Bartlett, ${ }^{6}$ M. A. Barton, ${ }^{6}$ I. Bartos, ${ }^{9}$ J. C. Batch, ${ }^{6}$ A. S. Bell, ${ }^{7}$ I. Belopolski, ${ }^{9}$ J. Bergman, ${ }^{6}$ J. Betzwieser, ${ }^{3}$ G. Billingsley, ${ }^{1}$ J. Birch, ${ }^{3}$ S. Biscans,${ }^{8}$ C. Biwer, ${ }^{5}$ E. Black, ${ }^{1}$ C. D. Blair, ${ }^{10}$ C. Bogan, ${ }^{11}$ C. Bond, ${ }^{34}$ R. Bork, ${ }^{1}$ D. O. Bridges, ${ }^{3}$ A.F. Brooks, ${ }^{1}$ D. D. Brown, ${ }^{34}$ L. Carbone, ${ }^{34}$ C. Celerier, ${ }^{12}$ G. Ciani, ${ }^{4}$ F. Clara, ${ }^{6}$ D. Cook, ${ }^{6}$ S. T. Countryman, ${ }^{9}$ M. J. Cowart, ${ }^{3}$ D. C. Coyne, ${ }^{1}$ A. Cumming, ${ }^{7}$ L. Cunningham, ${ }^{7}$ M. Damjanic, ${ }^{11}$ R. Dannenberg, ${ }^{1}$ K. Danzmann, ${ }^{13,11}$ C.F. Da Silva Costa, ${ }^{4}$ E. J. Daw, ${ }^{14}$ D. DeBra, ${ }^{12}$ R. T. DeRosa, ${ }^{3}$ R. DeSalvo, ${ }^{15}$ K. L. Dooley, ${ }^{16}$ S. Doravari, ${ }^{3}$ J. C. Driggers, ${ }^{6}$ S. E. Dwyer, ${ }^{6}$ A. Effler, ${ }^{3}$ T. Etzel,,${ }^{1}$ M. Evans, ${ }^{8}$ T. M. Evans, ${ }^{3}$ M. Factourovich, ${ }^{9}$ H. Fair ${ }^{5}$ D. Feldbaum, ${ }^{4,3}$ R. P. Fisher, ${ }^{5}$ S. Foley, ${ }^{8}$ M. Frede,${ }^{11}$ A. Freise,${ }^{34}$ P. Fritschel,${ }^{8}$ V. V. Frolov, ${ }^{3}$ P. Fulda, ${ }^{4}$ M. Fyffe, ${ }^{3}$ V. Galdi, ${ }^{15}$ J. A. Giaime, ${ }^{2,3}$ K. D. Giardina, ${ }^{3}$ J. R. Gleason, ${ }^{4}$ R. Goetz, ${ }^{4}$ S. Gras, ${ }^{8}$ C. Gray ${ }^{6}$ R. J. S. Greenhalgh, ${ }^{17}$ H. Grote, ${ }^{11}$ C. J. Guido, ${ }^{3}$ K. E. Gushwa, ${ }^{1}$ E. K. Gustafson, ${ }^{1}$ R. Gustafson, ${ }^{18}$ G. Hammond, ${ }^{7}$ J. Hanks, ${ }^{6}$ J. Hanson, ${ }^{3}$ T. Hardwick, ${ }^{2}$ G. M. Harry, ${ }^{19}$ K. Haughian, ${ }^{7}$ J. Heefner, ${ }^{1 *}$ M. C. Heintze, ${ }^{3}$ A. W. Heptonstall, ${ }^{1}$ D. Hoak,${ }^{20}$ J. Hough, ${ }^{7}$ A. Ivanov, ${ }^{1}$ K. Izumi, ${ }^{6}$ M. Jacobson, ${ }^{1}$ E. James, ${ }^{1}$ R. Jones, ${ }^{7}$ S. Kandhasamy, ${ }_{16}$ S. Karki,${ }^{21}$ M. Kasprzack, ${ }^{2}$ S. Kaufer, ${ }^{13}$ K. Kawabe, ${ }^{6}$ W. Kells, ${ }^{1}$ N. Kijbunchoo, ${ }^{6}$ E. J. King, ${ }^{22}$ P. J. King, ${ }^{6}$ D. L. Kinzel, ${ }^{3}$ J. S. Kissel,,${ }^{6}$ K. Kokeyama, ${ }^{2}$ W. Z. Korth, ${ }^{1}$ G. Kuehn, ${ }^{11}$ P. Kwee, ${ }^{8}$ M. Landry, ${ }^{6}$ B. Lantz, ${ }^{12}$ A. Le Roux, ${ }^{3}$ B. M. Levine, ${ }^{6}$ J. B. Lewis, ${ }^{1}$ V. Lhuillier, ${ }^{6}$ N. A. Lockerbie, ${ }^{23}$ M. Lormand, ${ }^{3}$ M. J. Lubinski, ${ }^{6}$ A. P. Lundgren, ${ }^{11}$ T. MacDonald,${ }^{12}$ M. MacInnis, ${ }^{8}$ D. M. Macleod, ${ }^{2}$ M. Mageswaran, ${ }^{1}$ K. Mailand, ${ }^{1}$ S. Márka, ${ }^{9}$ Z. Márka, ${ }^{9}$ A. S. Markosyan, ${ }^{12}$ E. Maros, ${ }^{1}$ I. W. Martin, ${ }^{7}$ R. M. Martin, ${ }^{4}$ J. N. Marx, ${ }^{1}$ K. Mason, ${ }^{8}$ T. J. Massinger, ${ }^{5}$ F. Matichard, ${ }^{8}$ N. Mavalvala, ${ }^{8}$ R. McCarthy, ${ }^{6}$ D. E. McClelland, ${ }^{24}$ S. McCormick, ${ }^{3}$ G. McIntyre, ${ }^{1}$ J. McIver, ${ }^{1}$ E. L. Merilh, ${ }^{6}$ M. S. Meyer, ${ }^{3}$ P. M. Meyers, ${ }^{25}$ J. Miller, ${ }^{8}$ R. Mittleman, ${ }^{8}$ G. Moreno, ${ }^{6}$ C. L. Mueller ${ }^{4}$ G. Mueller, ${ }^{4}$ A. Mullavey, ${ }^{3}$ J. Munch, ${ }^{22}$ P. G. Murray, ${ }^{7}$ L. K. Nuttall, ${ }^{5}$ J. Oberling, ${ }^{6}$ J. O?Dell, ${ }^{17}$ P. Oppermann, ${ }^{11}$ Richard J. Oram, ${ }^{3}$ B. O?Reilly, ${ }^{3}$ C. Osthelder, ${ }^{1}$ D. J. Ottaway, ${ }^{22}$ H. Overmier, ${ }^{3}$ J.R. Palamos, ${ }^{21}$ H. R. Paris, ${ }^{12}$ W. Parker, ${ }^{3}$ Z. Patrick, ${ }^{12}$ A. Pele, ${ }^{3}$ S. Penn ${ }^{26}$ M. Phelps, ${ }^{7}$ M. Pickenpack, ${ }^{11}$ V. Pierro, ${ }^{15}$ I. Pinto, ${ }^{15}$ J. Poeld, ${ }^{11}$ M. Principe, ${ }^{15}$ L. Prokhorov, ${ }^{27}$ O. Puncken, ${ }^{11}$ V. Quetschke, ${ }^{28}$ E. A. Quintero, ${ }_{1}^{1}$ F. J. Raab, ${ }^{6}$ H. Radkins, ${ }^{6}$ P. Raffai, ${ }^{29}$ C. R. Ramet, ${ }^{3}$ C. M. Reed, ${ }^{6}$ S. Reid ${ }^{30}$ D. H. Reitze, ${ }^{1,4}$ N. A. Robertson, ${ }^{1,7}$ J. G. Rollins, ${ }^{1}$ V. J. Roma, ${ }^{21}$ J. H. Romie, ${ }^{3}$ S. Rowan, ${ }^{7}$ K. Ryan, ${ }^{6}$ T. Sadecki, ${ }^{6}$ E. J. Sanchez, ${ }^{1}$ V. Sandberg, ${ }^{6}$ V. Sannibale, ${ }^{1}$ R. L. Savage, ${ }^{6}$ R. M. S. Schofield, ${ }^{21}$ B. Schultz, ${ }^{11}$ P. Schwinberg, ${ }^{6}$ D. Sellers, ${ }^{3}$ A. Sevigny, ${ }^{6}$ D. A. Shaddock, ${ }^{24}$ Z. Shao, ${ }^{1}$ B. Shapiro, ${ }^{12}$ P. Shawhan, ${ }^{31}$ D. H. Shoemaker, ${ }^{8}$ D. Sigg, ${ }^{6}$ B. J. J. Slagmolen, ${ }^{24}$ J. R. Smith, ${ }^{32}$ M. R. Smith, ${ }^{1}$ N. D. Smith-Lefebvre, ${ }^{1}$ B. Sorazu ${ }^{7}$ A. Staley, ${ }^{9}$ A. J. Stein, ${ }^{8}$ A. Stochino, ${ }^{1}$ K. A. Strain, ${ }^{7}$ R. Taylor, ${ }^{1}$ M. Thomas, ${ }^{3}$ P. Thomas, ${ }^{6}$ K. A. Thorne, ${ }^{3}$ E. Thrane ${ }^{33}$ K. V. Tokmakov, ${ }^{7}$ C. I. Torrie, ${ }^{1}$ G. Traylor, ${ }^{3}$ G. Vajente, ${ }^{1}$ G. Valdes ${ }^{28}$ A. A. van Veggel, ${ }^{7}$ M. Vargas,${ }^{3}$ A. Vecchio, ${ }^{34}$ P. J. Veitch, ${ }^{22}$ K. Venkateswara, ${ }^{35}$ T. Vo ${ }^{5}$ C. Vorvick, ${ }^{6}$ S. J. Waldman, ${ }^{8}$ M. Walker, ${ }^{2}$ R. L. Ward, ${ }^{24}$ J. Warner, ${ }^{6}$ B. Weaver, ${ }^{6}$ R. Weiss, ${ }^{8}$ T. Welborn, ${ }^{3}$ P. Weßels, ${ }^{11}$ C. Wilkinson, ${ }^{6}$ P. A. Willems, ${ }^{1}$ L. Williams, ${ }^{4}$ B. Willke, ${ }^{13,11}$ I. Wilmut, ${ }^{17}$ L. Winkelmann, ${ }^{11}$ C. C. Wipf, ${ }^{1}$ J. Worden, ${ }^{6}$ G. Wu, ${ }^{3}$ H. Yamamoto, ${ }^{1}$ C. C. Yancey, ${ }^{31}$ H. Yu, ${ }^{8}$ L. Zhang, ${ }^{1}$ M. E. Zucker, ${ }^{1,8}$ and J. Zweizig ${ }^{1}$

The author list has been corrected as of 8 March 2018. The author list is incorrect in the printed version of the journal. 